\title{
Myocyte Enhancer Factor-2 (MEF2) in Diseases of Central Nervous System: A Mini Review
}

\author{
Jing Wang* \\ Key Laboratory of Orthopedics of Gansu Province, the Second Hospital of Lanzhou University, Lanzhou, Gansu, China
}

\begin{abstract}
Myocyte enhancer factor-2 (MEF2), a family of transcription factors originally identified in muscles cells, was recently found to be expressed in the central nervous system. It is involved in the modulation of synapse elimination, a vital process that determines the fate and function of neurons. Recent studies suggested that MEF2 transcription factors are involved in synaptic plasticity, the molecular mechanism underlying learning and memory and other processes involved in dementia and neurodegenerative disease. This review summarizes recent advancements in understanding the role of MEF2 in neurodegenerative diseases, such as Alzheimer's disease and Parkinson's disease, and Fragile X syndrome.
\end{abstract}

\section{Introduction}

Diseases in the central nervous system (CNS) including neurodegenerative diseases are a group of diseases characterized by progressive loss of neuronal function in the brain, such as dementia (represented by Alzheimer's disease, AD) and Parkinson's disease (PD). In past decades, a growing body of evidence has suggested that neurodegenerative diseases originate with long-term changes in gene transcription in the central CNS, resulting in dysregulation of cellular energy homeostasis, transcription, translation, and folding of proteins.[1] Regulation of gene transcription and protein synthesis by transcription factors in the CNS plays vital roles in connecting intrinsic and extrinsic stimuli to protein production, the establishment of neuronal networks, and the formation and maintenance of long-term memo-

Keywords: Myocyte enhancer factor-2; Synapse elimination; Neurodegenerative disease.

Abbreviations: 6-OHDA, 6-hydroxydopamine; A $\beta$, amyloid $\beta$; AMPAR, $\alpha$-amino3-hydroxy-5-methyl-4-isoxazolepropionic acid receptor; Arc, activity-regulated cytoskeletal-associated protein; CNS, central nervous system; EPSC, excitatory postsynaptic current; FMRP, Fragile X Mental Retardation Protein; GR, glucocorticoid receptor; GSK3 $\beta$, glycogen synthase kinase 3beta; hAPP, human amyloid precursor protein; HIV, human immunodeficiency virus; IL-10, interleukin-10; KLF6, protein kinase A; PKA, Kruppel-like factor 6; MADS, minichromosome maintenance protein 1 (MCM1)-agamous-deficiens-serum response factors; MCM1, minichromosome maintenance protein 1; MEF2, myocyte enhancer factor-2; mGluR5, metabotropic glutamate receptor 5; MHCI, major histocompatibility complex class I; MPTP, 1-methyl-4-phenyl-1,2,3,6-tetrahydropyridine; NPC, neural stem/progenitor cells; p38MAPK, p38 mitogen-activated protein kinase; PSD-95, postsynaptic density protein-95; SNpc, substantia nigra pars compacta; TNF- $\alpha$, tumor necrosis factor- $\alpha$.

Received: 25 June 2015; Revised: 4 September 2015; Accepted: 14 October 2015 DOI: 10.14218/ERHM.2015.00001

*Correspondence to: Jing Wang, No. 82 Cui Ying Men St., Lanzhou, Gansu 730030, China. Tel: +86-136-09372860, Fax: +86-931-8942722, E-mail: wang_jing@1zu.edu. $\mathrm{cn}$ ries.[2]

Belonging to the minichromosome maintenance protein 1 (MCM1)-agamous-deficiens-serum response factors (MADS), myocyte enhancer factor-2 (MEF2) is a family of transcription factors that have been well-characterized in the development of multiple tissues. The role of MEF2 in muscles, where MEF2 was initially identified, has been well characterized.[3] Recent studies have indicated that the differentiation and maturation of neural stem/progenitor cells (NSCs) in conditional knockout of Mef2c mice were impaired and that $M e f 2 c$ conditional knockout mice exhibited behavioral abnormalities.[4] Given the diverse role of MEF2 in the CNS, it follows that MEF2 may be involved in AD, $\mathrm{PD}$, and Fragile $\mathrm{X}$ syndrome (FXS), through regulating synaptic plasticity[2,5] and N-methyl-D-aspartate receptors (NMDAR).[6]

\section{Expression of MEF2 family transcription factors in CNS}

The MEF2 family of transcription factors include four different nuclear proteins named MEF2A-D and was originally detected in muscle and later found to be differentially expressed in neurons of different regions of the brain. MEF2, either homodimers or heterodimers, can activate gene expression by binding as a co-factor to helix-loop-helix factors in specific tissues.[7-9] The N-terminal of MEF2 contains a highly conserved MADS-box, a motif that mediates dimerization, DNA binding, and interaction with co-factor, while the C-terminal sequence of MEF2 is variable and contains transcriptional activation domains that are targets of phosphorylation and other modifications.[8-10]

In the CNS, the four members of MEF2 are differentially expressed in the olfactory bulb, frontal cortex, cerebellum, hippocampus, thalamus, midbrain, and hindbrain during maturation of neurons.[11,12] In rat embryonic cortical neurons, MEF2A, MEF2C, and MEF2D mRNAs are all highly expressed, but MEF2 proteins are only encoded by the Mef2c gene.[13] MEF2D is developmentally expressed in proliferating neuronal cells and glial cells, i.e., MEF2D expression is increased in neurons following differentiation and growth and is decreased in mature glial cells.[14] All four members of MEF2 are expressed in apoptotic cerebellar granule neurons, but only MEF2A and MEF2D proteins were found to be phosphorylated.[15] Taken together, these results suggest that MEF2 is involved in the modulation and development of the CNS. In addition, MEF2D is expressed in microglia, and its level is increased in activated microglia by various in vivo and in vitro stimuli. MEF2D bound to the promoter of interleukin-10 (IL-10) increased its production and decreased the production of tumor necrosis factor- $\alpha$ (TNF- $\alpha$ ).[16] Therefore, MEF2 likely plays an important role in neurodegenerative diseases, as IL-10 and TNF- $\alpha$ are important factors in the pathology of neurodegenerative disease. 
MEF2, synapse elimination, and plasticity

The dynamic balance between synapse formation and synapse elimination is critical for the development and maturation of the brain throughout life in order to adapt to the intrinsic and extrinsic environment. Recent studies indicated that MEF2 plays a vital role in synapse elimination and is modulated by multiple molecules. Activation of MEF2 was shown to decrease synapse strength in cortical neurons via major histocompatibility complex class I (MHCI) and calcineurin[17] and to eliminate excitatory synapses in hippocampus.[18] Likewise, inhibition of endogenous MEF2 increased synapse number.[18] The effects of MEF2 activation and inhibition on synapse number were blocked in hippocampal neurons from Fragile $\mathrm{X}$ mental retardation gene $(F m r l)$ knockout mice, an animal model of FXS. These effects were restored by acute postsynaptic expression of the RNA-binding protein Fragile X Mental Retardation Protein (FMRP), encoded by Fmrl.[18] In vivo, knockdown of MEF2A suppressed expression of synaptoragmin-1 and eliminated synapses via a presynaptic mechanism.[19] In MEF knockout mice, the frequency of miniature excitatory postsynaptic currents (mEPSCs) in dentate gyrus was increased with no change in amplitude, and the paired-pulse ratio (ratio of $\mathrm{P} 2 / \mathrm{P} 1$ ) was decreased. Over-expression of $\mathrm{MEF} 2 \mathrm{C}$ decreased the frequency of mEPSCs.[20] Taken together, these studies suggest that MEF2 plays an important role in synapse elimination.

Several target genes have been reported to be involved in synapse elimination by MEF2. Knockdown of MEF2 was reported to reduce expression of the glucocorticoid receptor (GR) target gene c-jun in hippocampal culture.[21] The bidirectional modulation of synapse function in hippocampus by FMRP was also modulated by MEF2 activity, which was tuned by neuronal depolarization and potentiated during development.[22] In addition, MEF2 was shown to activate the RNA-binding protein FMRP and the autismspectrum disorder gene protocadherin $10(P c d h 10)$, decrease the level of eukaryotic translation elongation factor 1 alpha (EF1alpha), and induce degradation of postsynaptic density protein-95 (PSD-95), leading to synapse elimination.[23] In addition, in striatal medium spiny neurons cocultured with cortex, depolarization decreased synapse number in a MEF2-dependent manner via activation of the synapse remodeling genes Nur77 and activity-regulated cytoskeletal-associated protein (Arc, also known as Arg3.1).[24] Chan et al. found that MEF2D can be activated through phosphorylation by ataxia telangiectasia mutated (ATM) gene encoding kinase, which promoted the survival of cerebellar granule cells and was, thus, neuroprotective in ataxia-telangiectasia.[25] In addition, the gene encoding Arc was shown to be required for MEF2-induced synapse elimination.[26]

The effects of MEF2 on synapse elimination can be modulated by other factors. Arc is a protein targeted to neuronal dendrites, where it regulates MEF-induced synapse elimination. The expression of Arc is modulated by dendritic metabotropic glutamate receptor 5 (mGluR5) in hippocampal CA1 neurons,[26,27] suggesting that mGluR5 is involved in the modulation of MEF2-induced synapse elimination. In addition, dopamine receptors can change synapses number via MEF2. Cocaine increased synapse number in the striatum and induced phosphorylation of MEF2A, and this effect was attenuated by inhibition of D1 dopamine receptors but potentiated by inhibition of D3 receptors.[28] In delta-catenin knockdown mice, a model of mental retardation, expression of MEF2 promoted spine elimination in neurons.[29] Taken together, these studies on MEF2 in synapse elimination and plasticity suggest that MEF2 may play a critical role in neurodegenerative diseases.
MEF2 and neurodegenerative diseases

\section{MEF2 and dementia diseases}

Dementia diseases range in severity from mild cognitive impairment to $\mathrm{AD}$, which is characterized by the formation of amyloid $\beta(\mathrm{A} \beta)$ plaque in neurons. Wild-type human amyloid precursor protein (hAPP) can phosphorylate MEF2 via activation of p38 mitogen-activated protein kinase (p38MAPK), a modulator in $\mathrm{AD}$, and MEF2 may potentiate the anti-apoptotic effect of hAPP in neurons. [30] A genetic analysis of MEF2A in late-onset AD indicated that there was a correlation between the Pro279Leu mutation in exon 8 of MEF2 and late-onset AD,[31] suggesting that MEF2 is involved in the pathology of AD. Indeed, it was found that overexpression of MEF2 disrupted the formation of spatial memory specific to the dentate gyrus of mice and blocked the formation of long-term contextual and fear memory specific to lateral amygdala. Overexpression of MEF2 also blocked training-induced increases in neuronal spine density. In contrast, inhibition of MEF2 activity via its phosphorylation and downregulation of MEF2A and MEF2D proteins has been shown to promote memory formation. In addition, it was shown that artificially disturbing MEF2 function rescued the formation of memory by decreasing the expression of Arc and thereby increasing the surface expression of $\alpha$-amino-3-hydroxy-5-methyl-4-isoxazolepropionic acid receptors (AMPARs).[32] MEF2C knockout mice demonstrated impaired contextual and cue-dependent learning and memory, which are hippocampus-dependent.[20] These studies demonstrate a key role for MEF2 in learning and memory and support the hypothesis that MEF2 activity is involved in the pathology of AD. The major subunit of MEF2 involved in the learning and memory deficit is thought to be MEF2D because mice with MEF2A knockout alone did not show any changes in behavior, including learning and memory, and mice with MEF2A/2C double knockout only exhibited impaired motor coordination and enhanced release probability in hippocampal Schaffer collateral-CA1 synapses but no changes in learning and memory, long-term synaptic plasticity, or spine structure or density. However, triple MEF2A/2C/2D knockdown mice showed early postnatal lethality, increased neuronal apoptosis, and impaired short-term synaptic plasticity of Schaffer collateralCA1.[33] Glycogen synthase kinase 3beta (GSK3 $\beta$ ) phosphorylates MEF2D and is known to play a role in synaptic plasticity and neuronal death-related neurodegenerative diseases.[34] Therefore, MEF2D is likely neuroprotective, and dysregulation of MEF2 may contribute to the development of dementia diseases.

The mechanism of MEF2 in dementia diseases is related to its interaction with glutamate receptors. In dementia patients infected with human immunodeficiency virus (HIV), neuronal MEF2C is downregulated by NMDA-induced activation of microRNA-21.[35] NMDA-induced excitotoxicity in cortical neurons is considered a major cause of dementia diseases due to oxidative stress and in vivo focal stroke, leading to the activation of caspase-3/7 and subsequent cleavage of MEF2A, 2C, and 2D isoforms. Ultimately, these changes result in neuronal apoptosis, which can be prevented by transfection of constitutively active MEF2 (MEF2C-CA).[36] Excitotoxicity-induced activation of extrasynaptic NMDARs promoted calpain-mediated degradation of MEF2D, while knockdown of MEF2D ameliorated excitotoxicity induced by NMDA.[6] In the perforant-dentate gyrus synapses of MEF2 KO mice, the AMPAR and NMDAR EPSCs were increased.[20] MEF2C was shown to bind to the promoter of NMDAR subunit 1 (NR1).[37] White noise visual stimulation reduced MEF2A/2D and structural and developmental metaplas- 
Wang J.: MEF2 and diseases in central nervous system

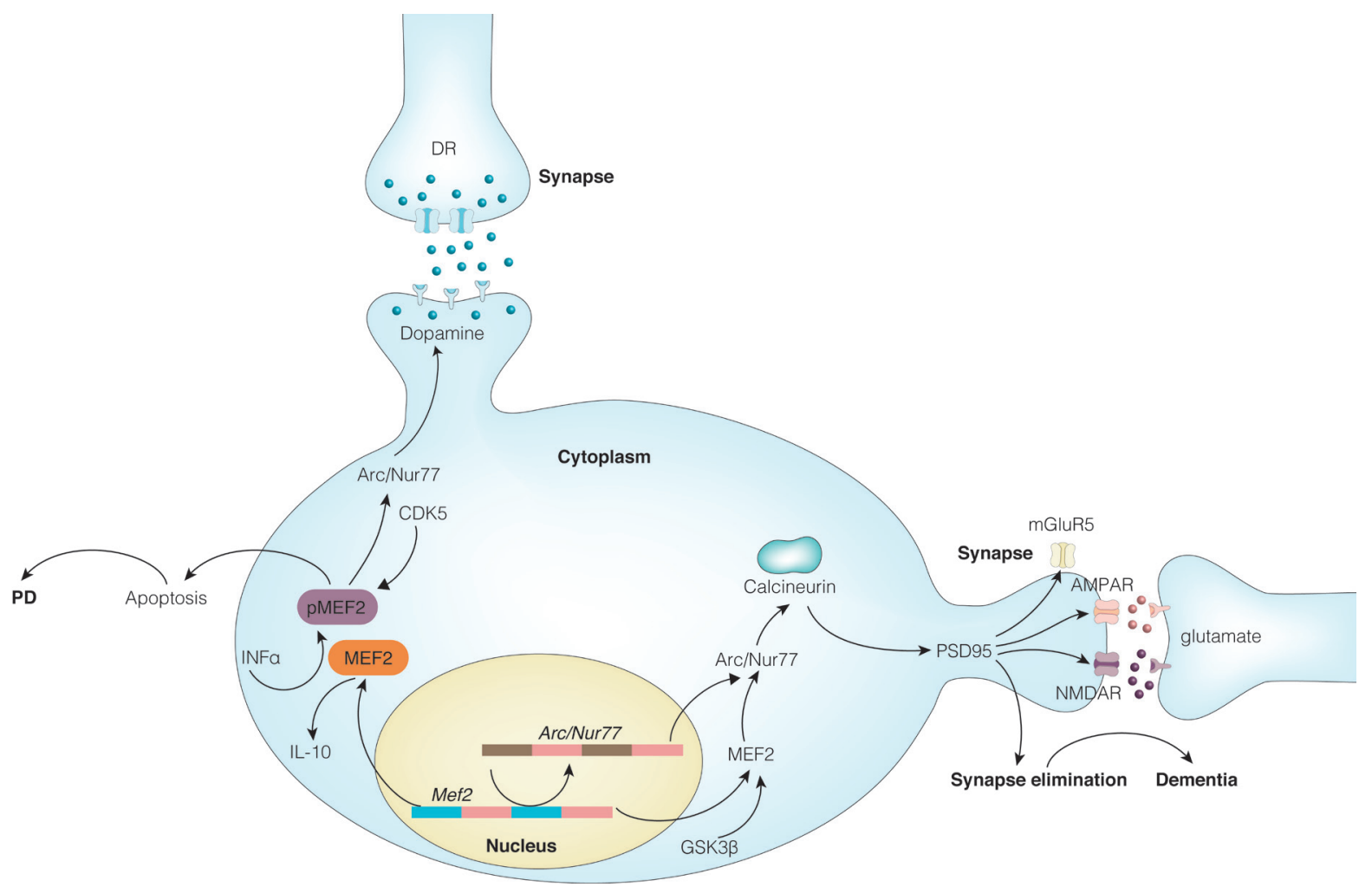

Fig. 1. A summary of the effects of MEF2 and the signaling pathways involved in synapse elimination and Parkinson's Disease.

ticity of neurons involving NDMAR and capsase-3/7/9.[38] The interaction between MEF2 and glutamate receptors may be mediated by Arc. Arc, a MEF2 target gene, is localized to neuronal dendrites where it regulates the processing and storage of information, synaptic plasticity, and learning/memory by modulating AMPAR endocytosis.[27] Furthermore, Arc activation of MEF2 as well as inhibition of phosphatidylinositol 3 kinase (PI3K)-AMPAR-GluA2 contributed to postoperative cognition disorder.[39] Taken together, these data suggest that MEF2 plays a role in both synaptic plasticity and dementia diseases via its interaction with glutamatergic receptors.

\section{MEF2 and fragile $X$ mental retardation}

FXS is the most common form of inherited mental retardation and autism and is caused by a mutation in the Fmrl gene, which encodes a non-functional RNA-binding protein, FMRP. The major symptoms/signs of FXS are hypersensitivity to sensory stimuli, stereotypic movements, social anxiety, hyperactivity, and severe impairment of cognition. FRMP has been shown to regulate synapse formation and maturation bidirectionally during development, i.e., facilitation in the first postnatal week and inhibition in the second postnatal week.[22] During development in Fmrl KO mice, the dendritic spine density in dentate gyrus is higher than wild-type mice,[40] suggesting a delay in synapse downregulation. However, MEF2-induced synapse elimination and the increase in synapses due to inhibition of MEF2 are blocked in hippocampal neurons of Fmr1 KO mice. These deficits can be recovered by acute postsynaptic expression of FMRP,[18] demonstrating that the postsynaptic interaction between MEF2 and FRMP is disturbed in FXS.
In the hippocampus, it was found that FMRP can bidirectionally regulate excitatory synaptic function in dendrites by binding to dendritic RNA, i.e., promoting synapse maturation at P6-7 while suppressing synapse formation at P13-16. FMRP expression was also modulated by MEF2 activity, which is, in turn, tuned by neuronal depolarization and is potentiated following the development.[22] In delta-catenin knockdown mice, which is a model of mental retardation, induction of MEF2 expression promoted spine elimination in neurons.[29] In FMRP-lacking neurons, the ubiquitination of PSD-95 induced by MEF2 was blocked,[23] while the expression of NMDAR subtypes (NR2A and NR2B) and PSD-95 were decreased.[41] These studies suggest a role of MEF2 in FXS through FMRP and glutamatergic receptors.

\section{MEF2 and Parkinson's diseases}

$\mathrm{PD}$ is due to the progressive and selective loss of dopaminergic neurons in the substantia nigra pars compacta $(\mathrm{SNpc})$ and the second most common neurodegenerative disease. Given the role of MEF2 in neuronal fate, it is possible it plays a role in the pathophysiology of PD. MEF2 was reported to be phosphorylated by GR activation and, therefore, can be switched from enhancer to repressor of transcription through c-JUN.[21] In primary hippocampal neuronal culture, activation of cyclic AMP (cAMP)/protein kinase A (PKA) signaling pathway inhibited trans-activation of MEF2D, which resulted in inhibition of the transcriptional target Kruppellike factor 6 (KLF6) and induced apoptosis.[42] Cocaine can reduce the activity of MEF2 through calmodulin and calcineurin signaling, promoting an increase in the density of dendritic spines on medium spiny neurons in the nucleus accumbens,[43] a region implicated in the pathology of PD. In addition, MEF2 activity was 
negatively regulated by CDK 5 and GSK $3 \beta$, kinases activated by toxic signals during PD pathology.[1] Phosphorylation of MEF2 by CDK 5 led to its inactivation and loss, resulting in inactivation of Nur77 and in vivo nigrostriatal degeneration,[44] a hallmark of PD. In striatal medium spiny neurons co-cultured with cortex, depolarization decreased synapse number in a MEF2-dependent manner through the synapse remodeling genes Nur77 and Arc. [24] Clinically, PD significantly decreased the level of MEF2D in the cytoplasm and nuclei of neurons in SNpc compared to the SNpc of age-matched controls and AD cases. In addition, oxidized MEF2D was increased in postmortem brains of PD patients.[45] These studies suggest that MEF2 is involved in PD pathology. Furthermore, MEF2D was found to increase microglial release of IL-10 and decrease TNF- $\alpha$ and, therefore, negatively regulate inflammation and inflammation-mediated cytotoxicity, important aspects of PD.[16] These studies suggest that MEF2D is involved in inflammation and oxidization processes during PD.

The role of MEF2 in PD is related to the activities of the dopamine system. Inhibition of MEF2D increased the toxicity of 1-methyl-4-phenyl-1,2,3,6-tetrahydropyridine (MPTP, a neurotoxin that destroys dopaminergic neurons and causes permanent symptoms of PD) and decreased the viability of SNc dopamine neurons.[46] Silencing MEF2C prevented the differentiation of hESC-derived neural stem/progenitor cells (NPCs) into dopaminergic neurons in vitro.[47] In contrast, overexpression of MEF2C facilitated the differentiation of dopaminergic neurons in vitro, improved the survival and differentiation of NPCs, and improved the PD signs in a rat model of PD induced by 6-hydroxydopamine (6-OHDA).[47] Furthermore, 6-OHDA was shown to oxidize MEF2D and increase the expression of the receptor for chaperone-mediated autophagy in the SNpc of mouse. Oxidized MEF2D was shown to be bound to heat-shock cognate protein $70 \mathrm{kDa}(\mathrm{Hsc} 70)$ in the dopamine neuronal cell line SN4741, which caused cell death.[45] The modulation of cocaine-induced phosphorylation of MEF2A by dopaminergic activity is also consistent with a role for MEF2 in PD pathology.[28]

\section{Prospective and prediction}

The prevalence of neurodegenerative diseases like PD and AD continues to increase in modern society. However, no ideal therapeutic and preventive strategies are available. Most neurodegenerative diseases are associated with memory impairment due to the disturbances in synaptic plasticity. Considering the role of MEF2 in synaptic plasticity and synapse elimination,[2] it is very possible that MEF2 plays an important role in the pathology of neurodegenerative diseases. It is our working hypothesis that the disturbance of MEF2 activity by congenital and acquired factors creates an imbalance in synapse dynamics, altering the fate of neurons and resulting in the onset of neurodegenerative disease. Following the clarification of the mechanisms underlying MEF2 in neurodegenerative diseases, some therapeutic strategies targeting MEF2 may include upstream and downstream signals, which will benefit the large number of patients suffering from neurodegenerative diseases.

\section{Conclusions}

MEF2 is a group of transcription factors playing multiple roles in different tissues. Recently studies indicated that MEF2 can modulate the functions of CNS through changing the activities of synapses such as synapse elimination and further determining the fate of neurons. Thus, MEF2 is identified to be involved in the pathology of some disorders in CNS such as AD, PD and Autism through multiple signal pathways, as shown in schematic Fig. 1. Following the clarification of the mechanisms of MEF2 in these disorders of CNS, it will be one target for the therapy of these disorders in future.

\section{Acknowledgements}

The study was supported by the National Natural Science Foundation of China (No. 81371230) and the National Science Foundation for Distinguished Young Scholars of Gansu Province, China (No. 1210RJDA010).

\section{Conflict of interest}

None.

\section{Author contributions}

Writing the paper (JW)

\section{References}

[1] Yin Y, She H, Li W, Yang Q, Guo S, Mao Z. Modulation of Neuronal Survival Factor MEF2 by Kinases in Parkinson's Disease. Front Physiol 2012;3:171. doi 10.3389/fphys.2012.00171

[2] Rashid AJ, Cole CJ, Josselyn SA. Emerging roles for MEF2 transcription factors in memory. Genes Brain Behav 2014;13(1):118-25. doi: 10.1111/gbb.12058.

[3] Naya FJ, Olson E. MEF2: a transcriptional target for signaling pathways controlling skeletal muscle growth and differentiation. Curr Opin Cell Biol 1999;11(6):683-8. doi: 10.1016/S0955-0674(99)00036-8.

[4] Li H, Radford JC, Ragusa MJ, Shea K, McKercher SR, Zaremba JD, et al. Transcription factor MEF2C influences neural stem/progenitor cell differentiation and maturation in vivo. Proc Natl Acad Sci USA 2008;105(27):9397-402. doi: 10.1073/pnas.0802876105.

[5] She H, Mao Z. Regulation of myocyte enhancer factor-2 transcription factors by neurotoxins. Neurotoxicology 2011;32(5):563-6. doi: 10.1016/j.neuro.2011.05.019

[6] Wei G, Yin Y, Li W, Bito H, She H, Mao Z. Calpain-mediated degradation of myocyte enhancer factor $2 \mathrm{D}$ contributes to excitotoxicity by activation of extrasynaptic N-methyl-D-aspartate receptors. J Biol Chem 2012;287(8):5797-805. doi: $10.1074 /$ jbc.M111.260109.

[7] Brand NJ. Myocyte enhancer factor 2 (MEF2). Int J Biochem Cell Biol 1997;29(12):1467-70. doi: 10.1016/S1357-2725(97)00084-8.

[8] Potthoff MJ, Olson EN. MEF2: a central regulator of diverse developmental programs. Development 2007;134(23):4131-40. doi: 10.1242/dev.008367.

[9] Dietrich JB. The MEF2 family and the brain: from molecules to memory. Cell Tissue Res 2013;352(2):179-90. doi: 10.1007/s00441-013-1565-2.

[10] Black B, Cripps R. Myocyte Enhancer Factor 2 Transcription Factors in Heart Development and Disease. In: Nadia Rosenthal, Richard P. Harvey, eds. Heart Development and Regeneration. Elsevier, 2010:673-99.

[11] Lyons GE, Micales BK, Schwarz J, Martin JF, Olson EN. Expression of mef2 genes in the mouse central nervous system suggests a role in neuronal maturation. J Neurosci 1995;15(8):5727-38.

[12] Lin X, Shah S, Bulleit RF. The expression of MEF2 genes is implicated in CNS neuronal differentiation. Brain Res Mol Brain Res 1996;42(2):307-16. doi: 10.1016/S0169-328X(96)00135-0.

[13] Lyons MR, Schwarz CM, West AE. Members of the myocyte enhancer factor 2 transcription factor family differentially regulate Bdnf transcription in response to neuronal depolarization. J Neurosci 2012;32(37):12780-5. doi: 10.1523/ JNEUROSCI.0534-12.2012.

[14] Lam BY, Chawla S. MEF2D expression increases during neuronal differentiation of neural progenitor cells and correlates with neurite length. Neurosci Lett 2007;427(3):153-8. doi: 10.1016/j.neulet.2007.09.030.

[15] Li M, Linseman DA, Allen MP. Myocyte enhancer factor 2A and 2D undergo phosphorylation and caspase-mediated degradation during apoptosis of rat cer- 
ebellar granule neurons. J Neurosci 2001;21(17):6544-52.

[16] Yang S, Gao L, Lu F, Wang B, Gao F, Zhu G, et al. Transcription factor myocyte enhancer factor $2 \mathrm{D}$ regulates interleukin-10 production in microglia to protect neuronal cells from inflammation-induced death. J Neuroinflammation $2015 ; 12(1): 258$

[17] Elmer BM, Estes ML, Barrow SL, McAllister AK. MHCI requires MEF2 transcription factors to negatively regulate synapse density during development and in disease. J Neurosci 2013;33(34):13791-804. doi: 10.1523/JNEUROSCI.2366-13.2013.

[18] Pfeiffer BE, Zang T, Wilkerson JR, Taniguchi M, Maksimova MA, Smith LN, et al. Fragile $\mathrm{X}$ mental retardation protein is required for synapse elimination by the activity-dependent transcription factor MEF2. Neuron 2010;66(2):191-7. doi: 10.1016/j.neuron.2010.03.017.

[19] Yamada T, Yang Y, Huang J, Coppola G, Geschwind DH, Bonni A. Sumoylated MEF2A coordinately eliminates orphan presynaptic sites and promotes maturation of presynaptic boutons. J Neurosci 2013;33(11):4726-40. doi: 10.1523/ JNEUROSCI.4191-12.2013.

[20] Barbosa AC, Kim MS, Ertunc M, Adachi M, Nelson ED, McAnally J, et al. $\mathrm{MEF} 2 \mathrm{C}$, a transcription factor that facilitates learning and memory by negative regulation of synapse numbers and function. Proc Natl Acad Sci USA 2008;105(27):9391-6. doi: 10.1073/pnas.0802679105.

[21] Speksnijder N, Christensen KV, Didriksen M, De Kloet ER, Datson NA. Glucocorticoid receptor and myocyte enhancer factor 2 cooperate to regulate the expression of c-JUN in a neuronal context. J Mol Neurosci 2012;48(1):209-18. doi: 10.1007/s12031-012-9809-2.

[22] Zang T, Maksimova MA, Cowan CW, Bassel-Duby R, Olson EN, Huber KM. Postsynaptic FMRP bidirectionally regulates excitatory synapses as a function of developmental age and MEF2 activity. Mol Cell Neurosci 2013;56:39-49. doi: 10.1016/j.men.2013.03.002

[23] Tsai NP, Wilkerson JR, Guo W, Maksimova MA, DeMartino GN, Cowan CW, et al. Multiple autism-linked genes mediate synapse elimination via proteasomal degradation of a synaptic scaffold PSD-95. Cell 2012;151(7):1581-94. doi: 10.1016/j.cell.2012.11.040

[24] Tian X, Kai L, Hockberger PE, Wokosin DL, Surmeier DJ. MEF-2 regulates activity-dependent spine loss in striatopallidal medium spiny neurons. Mol Cell Neurosci 2010;44(1):94-108. doi: 10.1016/j.men.2010.01.012.

[25] Chan SF, Sances S, Brill LM, Okamoto S, Zaidi R, McKercher SR, et al. ATM-dependent phosphorylation of MEF2D promotes neuronal survival af ter DNA damage. J Neurosci 2014;34(13):4640-53. doi: 10.1523/JNEUROSCI.2510-12.2014

[26] Wilkerson JR, Tsai NP, Maksimova MA, Wu H, Cabalo NP, Loerwald KW, et al. A role for dendritic mGluR5-mediated local translation of Arc/Arg3.1 in MEF2dependent synapse elimination. Cell Rep 2014;7(5):1589-600. doi: 10.1016/j. celrep.2014.04.035.

[27] Chowdhury S, Shepherd JD, Okuno H, Lydord G, Petralia RS, Plath N, et al. Arc/Arg3.1 interacts with the endocytic machinery to regulate AMPA receptor trafficking. Neuron 2006;52(3):445-59. doi: 10.1016/j.neuron.2006.08.033.

[28] Zhang L, Li J, Liu N, Wang B, Gu J, Zhang M, et al. Signaling via dopamine D1 and D3 receptors oppositely regulates cocaine-induced structural remodeling of dendrites and spines. Neurosignals 2012;20(1):15-34. doi: 10.1159/000330743.

[29] Yuan Y, Singh D, Arikkath J. Mef2 promotes spine elimination in absence of delta-catenin. Neurosci Lett 2013;536:10-13. doi: 10.1016/j.neulet.2013.01.002.

[30] Burton TR, Dibrov A, Kashour T, Amara FM. Anti-apoptotic wild-type Alzheimer amyloid precursor protein signaling involves the p38 mitogen-activated protein kinase/MEF2 pathway. Brain Res Mol Brain Res 2002;108(1-2):10220. doi: 10.1016/S0169-328X(02)00519-3.

[31] Gonzalez P, Alvarez V, Menendez M, Lahoz CH, Martinez C, Corao AI, et al. Myocyte enhancing factor-2A in Alzheimer's disease: genetic analysis and as- sociation with MEF2A-polymorphisms. Neurosci Lett 2007;411(1):47-51. doi: 10.1016/j.neulet.2006.09.055.

[32] Cole CJ, Mercaldo V, Restivo L, Yiu AP, Sekeres MJ, Han JH, et al. MEF2 negatively regulates learning-induced structural plasticity and memory formation. Nat Neurosci 2012;15(9):1255-64. doi: 10.1038/nn.3189.

[33] Akhtar MW, Kim MS, Adachi M, Morris MJ, Qi X, Richardson JA, et al. In vivo analysis of MEF2 transcription factors in synapse regulation and neuronal survival. PLoS One 2012;7(4):e34863. doi: 10.1371/journal.pone.0034863.

[34] Wang X, She H, Mao Z. Phosphorylation of neuronal survival factor MEF2D by glycogen synthase kinase 3beta in neuronal apoptosis. J Biol Chem 2009;284(47):32619-26. doi: 10.1074/jbc.M109.067785.

[35] Yelamanchili SV, Chaudhuri AD, Chen LN, Xiong H, Fox HS. MicroRNA-21 dysregulates the expression of MEF2C in neurons in monkey and human SIV/HIV neurological disease. Cell Death Dis 2010;1:e77. doi: 10.1038/cddis. 2010.56 .

[36] Okamoto S, Li Z, Ju C, Scholzke MN, Mathews E, Cui J, et al. Dominantinterfering forms of MEF2 generated by caspase cleavage contribute to NMDAinduced neuronal apoptosis. Proc Natl Acad Sci USA 2002;99(6):3974-9. doi: 10.1073 pnas.022036399.

[37] Krainc D, Bai G, Okamoto S, Carles M, Kusiak JW, Brent RN, et al. Synergistic activation of the N-methyl-D-aspartate receptor subunit 1 promoter by myocyte enhancer factor 2C and Sp1. J Biol Chem 1998;273(40):26218-24. doi: $10.1074 / \mathrm{jbc} .273 .40 .26218$

[38] Chen SX, Cherry A, Tari PK, Podgorski K, Kwong YK, Haas K. The transcription factor MEF2 directs developmental visually driven functional and structural metaplasticity. Cell 2012;151(1):41-55. doi: 10.1016/j.cell.2012.08.028.

[39] Hu N, Wang M, Xie K, Wang H, Wang C, Wang C, et al. Internalization of GluA2 and the underlying mechanisms of cognitive decline in aged rats following surgery and prolonged exposure to sevoflurane. Neurotoxicology 2015;49:94-103, doi: 10.1016/j.neuro. 2015.05.010.

[40] Grossman AW, Aldridge GM, Lee KJ, Zeman MK, Jun CS, Azam HS, et al. Developmental characteristics of dendritic spines in the dentate gyrus of Fmr1 knockout mice. Brain Res 2010;1355:221-7. doi: 10.1016/j.brainres.2010.07.090.

[41] Krueger DD, Osterweil EK, Chen SP, Tye LD, Bear MF. Cognitive dysfunction and prefrontal synaptic abnormalities in a mouse model of fragile $\mathrm{X}$ syndrome. Proc Natl Acad Sci USA 2011;108(6):2587-92. doi: 10.1073/pnas.1013855108.

[42] Salma J, McDermott JC. Suppression of a MEF2-KLF6 survival pathway by PKA signaling promotes apoptosis in embryonic hippocampal neurons. J Neurosci 2012;32(8):2790-803. doi: 10.1523/JNEUROSCI.3609-11.2012.

[43] Pulipparacharuvil S, Renthal W, Hale CF, Taniguchi M, Xiao G, Kumar A, et al. Cocaine regulates MEF2 to control synaptic and behavioral plasticity. Neuron 2008;59(4):621-33. doi: 10.1016/j.neuron.2008.06.020.

[44] Mount MP, Zhang Y, Amini M, Callaghan S, Kulczychi J, Mao Z, et al. Perturbation of transcription factor Nur77 expression mediated by myocyte enhancer factor 2D (MEF2D) regulates dopaminergic neuron loss in response to 1-methyl-4-phenyl-1,2,3,6-tetrahydropyridine (MPTP). J Biol Chem 2013;288(20):14362-71. doi: 10.1074/jbc.M112.439216.

[45] Gao L, She H, Li W, Zeng J, Zhu J, Jones DP, et al. Oxidation of survival factor MEF2D in neuronal death and Parkinson's disease. Antioxid Redox Signal 2014;20(18):2936-48. doi: 10.1089/ars.2013.5399.

[46] Wang B, Cai Z, Lu F, Li C, Zhu X, Su L, et al. Destabilization of survival factor MEF2D mRNA by neurotoxin in models of Parkinson's disease. J Neurochem 2014;130(5):720-8. doi: 10.1111/jnc. 12765.

[47] Cho EG, Zaremba JD, McKercher SR, Talantova M, Tu S, Masliah E, et al. MEF2C enhances dopaminergic neuron differentiation of human embryonic stem cells in a parkinsonian rat model. PLoS One 2011;6(8):e24027. doi: 10.1371/journal.pone.0024027. 